39. Z. Zhu et al., J. Virol. 85, 11401 (2011).

40. R. Diskin et al., Science 334, 1289 (2011).

41. Materials and methods are available as supplementary materials on Science Online.

42. One of the features of our approach is its ability to distinguish between antibodies targeting overlapping epitopes in a substantially different ways: There is a significant difference in the correlation coefficients for antibodies targeting a similar epitope versus the correlation coefficients for antibodies targeting different epitopes on the same site of vulnerability. Similarly, there is a significant difference for similar epitopes versus different sites of vulnerability; however, there is no significant difference for different epitopes on the same site of vulnerability versus different sites of vulnerability (fig. S1).

43. R. M. Cardoso et al., Immunity 22, 163 (2005).

44. Residue numbering throughout the paper is relative to strain HXB2, unless stated otherwise.

45. K. J. Doores et al., Proc. Natl. Acad. Sci. U.S.A. 107 17107 (2010).

46. Y. Li et al., J. Virol. 86, 11231 (2012).

47. To resolve the discrepancy between epitope mapping and neutralization-based predictions, we determined the cocrystal structure of VRCO6 in complex with the gp120 core and compared it with the structure of the VRC01-like antibody VRC03: The antibody variable-domain root mean square deviation upon alignment of the respective VRC06- and VRC03-bound gp120 cores was $1.45 \AA$ confirming the similarity in the modes of gp120 recognition by these two antibodies (Fig. $2 \mathrm{~B}$ and table S3). Although VRCO3 and VRC06 exhibit extraordinary similarity in their mode of recognition of gp120 when compared with VRC01, unlike VRC01, antibodies VRC03 and VRCO6 contain a long insertion in the heavy-chain framework 3 region that falls in the vicinity of the gp120 bridging sheet. In the crystallized complexes of these antibodies and monomeric gp120 core (which lacks the full V1/V2 region), the insertions do not make substantial contact with gp120. On the functional trimer spike, however, these insertions likely make additional contacts with the antigen, extending the antibody epitopes as compared with VRC01. The result is that VRCO3 and VRCO6 cluster in the periphery of the VRC01 cluster.

48. The division into monoclonal antibody clusters was based on the clustering results from the larger 34-strain panel (Fig. 2A), whereas serum delineation was performed on an available 21-strain panel (because of serum volume constraints, we used a 21-strain subset of the main panel). Although antibody clustering is sensitive to the size of the viral panel (appendix S1), predictions with smaller panels (such as the 21-strain panel used in the serum analysis) could nonetheless be sufficient (fig. S3) delineation of component-antibody specificities for the CAP256 sera was based on a smaller 13-strain panel (41).

49. A signal for PGT128-like antibodies in donor 45 was also observed. However, only part of the neutralization activity of the donor 45 serum could be attributed to VRC01-like antibodies (fig. S11), which indicated that other antibody specificities may also exist in that serum. It is thus not surprising to observe other neutralization signals in the predictions.

50. P. L. Moore et al., J. Virol. 85, 3128 (2011).

51. L. M. Walker et al., Proc. Natl. Acad. Sci. U.S.A. 108, 20125 (2011).

52. Sera were from clade B-infected donors from the cohort described in (28) with moderate to broad neutralization on the panel of 21 viruses.

53. T. Zhou et al., Retrovirology 9 (Suppl. 2), P57 (2012).

54. For example, standard serum mapping for glycan-V3 antibodies uses N332 mutants and, thus, may not be as sensitive for some antibodies in the PGT128-like group that are more affected by glycan-301 (table S4).

55. The neutralization-based delineation showed relatively strong signals $(>0.2)$ for 2G12- or b12-like antibodies with many of the sera, and potential VRC01-, PG9-, PGT128-, and 10E8-like specificities were predicted for specific subsets of the sera. In the case of 2G12-like activity, many serum samples were also found to be positive by experimental mapping assays, which indicated that this epitope might be a more frequent target compared with other major epitopes, although not necessarily by antibodies having the specific domain-exchanged nature of 2G12 (Fig. 3D). A clear threshold for the presence or absence of a particular specificity in a given serum was not apparent: A level of 0.35 positively identified 10E8-like antibodies in donor N152, but sporadic signals of up to $\sim 0.3$ were observed in longitudinal samples (Fig. 3B).

56. To confirm the predicted similarity to VRCO1, we crystallized and determined the structure of the antigen-binding fragment (Fab) of VRC23 in complex with an HIV-1 core gp120. The epitope footprints on gp120 for VRC23 and VRC01 were similar, with buried gp120 surface areas correlating significantly (Spearman $R=0.76, P<0.0001$ ) (Fig. $4 \mathrm{~A}$ and figs. S8 to S10)

57. M. D. Simek et al., J. Virol. 83, 7337 (2009).

58. The current analysis is validated primarily for a single major component-antibody specificity in serum, with predictions on other specificities yet to be ascertained; however, the ability to extract major specificities from polyclonal sera will likely be of considerable utility. Nevertheless, the observation that antibody neutralization fingerprints can capture sufficient information to allow, at least in some cases, the deconvolution of the polyclonal serum-based pattern of HIV-1 neutralization into neutralization from component antibodies is subject to a number of assumptions and limitations (41)

59. J. M. Binley et al., J. Virol. 78, 13232 (2004).

60. M. S. Seaman et al., J. Virol. 84, 1439 (2010).

61. Q. J. Sattentau, A. J. McMichael, F1000 Biol. Rep. 2, 60 (2010).

62. F. Klein et al., J. Exp. Med. 209, 1469 (2012)

Acknowledgments: Support for this work was provided by the Intramural Research Program of the Vaccine Research Center, NIAID, NIH, and by the International AIDS Vaccine Initiative. The CAPRISA cohort was established through grants from NIAID, NIH (U19 Al51794) and the South African Department of Science and Technology. The authors are grateful to ]. Stuckey for assistance with graphics and thank B. Korber, D. Montefiori, and M. Roederer and members of the Structural Biology Section, Structural Bioinformatics Core, Humoral Immunology Section, and Humoral Immunology Core at the NIH Vaccine Research Center for helpful discussions or comments on the manuscript. We thank ]. Baalwa, D. Ellenberger, D. Gabuzda, F. Gao, B. Hahn, K. Hong, J. Kim, F. McCutchan, D. Montefiori, L. Morris, ]. Overbaugh, E. Sanders-Buell, G. Shaw, R. Swanstrom, M. Thomson, S. Tovanabutra, C. Williamson, and L. Zhang for contributing the HIV-1 Envelope plasmids used in our neutralization panels. The data presented in this manuscript are tabulated in the main paper and in the supplementary materials. The NIH has filed a patent relating to neutralizing antibodies in this manuscript: WIPO no. WO 2011/038290 A2, titled "Neutralizing antibodies to HIV-1 and their use," with inventors ].R.M., P.D.K., M.C., L.S., and G.O. Structure factors and coordinates for the VRCO6 and VRC23 antibody cocrystal structures with HIV-1 gp120 have been deposited with the Protein Data Bank under accession codes 4]B9 and 4]6R, respectively. Scripts for the neutralization-based antibody clustering and serologic analysis are included in the online supplementary material and methods (appendix S2) and are available from I.S.G or P.D.K. upon request. Use of sector 22 (Southeast Region Collaborative Access team) at the Advanced Photon Source was supported by the U.S. Department of Energy, Basic Energy Sciences, Office of Science, under contract no. W-31-109-Eng-38.

Supplementary Materials

www.sciencemag.org/cgi/content/full/340/6133/751/DC1 Materials and Methods

Supplementary Text

Figs. S1 to S15

Tables S1 to S10

References (63-84)

12 December 2012; accepted 28 March 2013

$10.1126 /$ science.1233989

\title{
Emergence of Individuality in Genetically Identical Mice
}

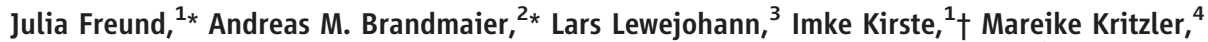
Antonio Krüger, ${ }^{4,5}$ Norbert Sachser, ${ }^{3}$ Ulman Lindenberger, ${ }^{2}$ Gerd Kempermann ${ }^{1,6} \ddagger$

Brain plasticity as a neurobiological reflection of individuality is difficult to capture in animal models. Inspired by behavioral-genetic investigations of human monozygotic twins reared together, we obtained dense longitudinal activity data on 40 inbred mice living in one large enriched environment. The exploratory activity of the mice diverged over time, resulting in increasing individual differences with advancing age. Individual differences in cumulative roaming entropy, indicating the active coverage of territory, correlated positively with individual differences in adult hippocampal neurogenesis. Our results show that factors unfolding or emerging during development contribute to individual differences in structural brain plasticity and behavior. The paradigm introduced here serves as an animal model for identifying mechanisms of plasticity underlying nonshared environmental contributions to individual differences in behavior.

$\mathrm{P}$ lasticity, or the reciprocal interaction between brain structure and function, draws on genetic and nongenetic sources of variation and forms the neurobiological basis of individuality. Behavioral-genetic studies with humans provide statistical tools for estimating the additive and interactive contributions of genetic and environmental variations to individual differences in behavioral development (1). In the case of monozygotic twins reared together, sibling differences reflect the influence of individual responses, based on the same genetic makeup, to a nominally identical environment. Somewhat paradoxically, this source of variation is generally referred to as the "nonshared environment" in behavior genetics. As Turkheimer has noted, "exactly what the nonshared environment consists of has been a matter of mystery and controversy for some time" [(2) p. 826]. We developed an animal model for studying the nonshared environment and examined its effects on behavioral and neural development.

In rodents, enriched environments are among the tools of choice for addressing the influence of a given environment on individuals with identical genetic background $(3,4)$. However, with some exceptions $[(5)$, see also $(3,6)]$ the emergence of experience-based individual differences within groups of genetically identical animals exposed 
to the same enriched environment has rarely been addressed. We used a large group of animals and a particularly complex environment to capture the emergence of individual differences in brain and behavior over time. We used exploration as a marker of behavioral development, and adult neurogenesis in the hippocampus as a marker for continued brain development.

Adult hippocampal neurogenesis allows lifelong plastic adaptation of the hippocampal neural network in the face of environmental complexity and novelty, is regulated by physical and cognitive aspects of behavioral activity, and can be quantified in a straightforward way and captured with numerical key parameters (7). We used the individual regulation of adult hippocampal neurogenesis in response to individual differences in experiencing a nominally identical environment as a proxy for individual brain plasticity. What happens when genetically identical mice inhabit the same environment? How much individuality emerges over time, and is it related to adult hippocampal neurogenesis? Do initial individual differences in behavior dominate later individual differences, or will novel variance emerge?

Forty female inbred mice (C57BL/6N), 4 weeks old at the beginning of the experiments, were kept in a large enriched environment (ENR) for

${ }^{1}$ CRTD-DFG Research Center for Regenerative Therapies Dresden, Technische Universität Dresden, 01307 Dresden, Germany. ${ }^{2}$ Center for Lifespan Psychology, Max Planck Institute for Human Development, 14195 Berlin, Germany. ${ }^{3}$ Department of Behavioural Biology, Westfälische Wilhelms-Universität Münster, 48149 Münster, Germany. ${ }^{4}$ Institute for Geoinformatics, Westfälische Wilhelms-Universität Münster, 48151 Münster, Germany. ${ }^{5}$ German Research Center for Artificial Intelligence, 66123 Saarbrücken, Germany. ${ }^{6}$ German Center for Neurodegenerative Disease (DZNE) Dresden, 01307 Dresden, Germany.

*These authors contributed equally to this work.

†Present address: Duke Cardiovascular Research Center and Mandel Center for Hypertension and Atherosclerosis Research, Duke University Medical Center, Durham, NC 27710, USA. ‡Corresponding author. E-mail: gerd.kempermann@dzne. de or gerd.kempermann@crt-dresden.de
3 months [(8); see Fig. 1A and detailed description in the supplementary materials]. The mice were tagged with radio-frequency identification (RFID) transponders, and 20 antennas, distributed over the entire environment, monitored their current locations.

Over the experimental period of 3 months (Fig. 1B), we saw an increase in mean body weight (Fig. 1C) compared with the baseline group $(N=8)$. Besides this age effect, variability of both body and brain weight (Fig. 1D) seemed larger in ENR mice than in control (CTR) mice at the end of the experiment (but $P=0.057$ for brain-weight variance, Bartlett-box $F$ test, $F_{11,39}=0.338$; and $P=$ 0.154 for body-weight variance, $F_{11,39}=0.449$ ).

Adult neurogenesis was assessed at the end of the experiment by counting proliferating precursor cells that had been labeled with bromodeoxyuridine (BrdU) 3 weeks before (Fig. 1B). Average adult neurogenesis was increased in the ENR group compared with the CTR group (Fig. 2A), decreasing the physiological age-related decline in adult neurogenesis (as shown in the comparison with the baseline value at 8 weeks). In line with previous studies, ENR animals also had significantly more new astrocytes than CTR animals $(t=4.321, P=0.0002)$ but there was no significant difference in cells with undefined phenotype ( $t=0.736, P=0.5$ ) (fig. S1) (9).

New neurons in the hippocampus are assumed to enable the hippocampus to flexibly cope with novelty and complexity (10). Physical activity and locomotion provide subjective proxy feedback signals that tend to indicate situations potentially rich in cognitive challenges requiring plasticity $(11,12)$. Our specific hypothesis was that mice with a greater range of experiences, as reflected in more explorative behavior corresponding to a larger and more intensive coverage of the territory, would show higher levels of adult hippocampal neurogenesis. Within the ENR group, individual differences in the total number of antenna contacts as proxy for the sheer amount of locomotion were not associated with individual differences in neurogenesis [correlation coefficient $(r)=0.133 ; t=0.829 ; P=0.412]$ (fig. S2) (13). Hence, we sought to identify a trait marker of activity that would be more likely to be associated with adult neurogenesis.

To obtain an ethologically valid index of explorative behavior, we derived a new measure, called "roaming entropy" (RE; for more details, see methods in the supplementary materials). RE is the entropy of the probability distribution of finding a mouse at a given antenna at a given time and, thus, is an indicator of the territorial range covered by a given mouse in a given period of time. $R E$ is a continuous measure. $\mathrm{RE}$ is low when a mouse has a stable and small home range, independent of the amount of locomotion within that area. But even a relatively large range can be covered with low RE if few stable spots of attendance are spread out over larger distances. RE is high, in contrast, if coverage is evenly distributed over the entire area of the cage (Fig. 2B; see also movies S1 and S2).

Measurements of RE were aggregated into four adjacent time periods (T1, T2, T3, and T4), each representing the average RE over 24 calendar days, and summed over time periods to obtain an index of cumulative roaming entropy (cRE; i.e., $\mathrm{cRE}_{\mathrm{T} 1}=\mathrm{RE}_{\mathrm{T} 1} ; \mathrm{cRE}_{\mathrm{T} 2}=\mathrm{cRE}_{\mathrm{T} 1}+\mathrm{RE}_{\mathrm{T} 2}$; $\left.\mathrm{cRE}_{\mathrm{T} 3}=\mathrm{cRE}_{\mathrm{T} 2}+\mathrm{RE}_{\mathrm{T} 3} ; \mathrm{cRE}_{\mathrm{T} 4}=\mathrm{cRE}_{\mathrm{T} 3}+\mathrm{RE}_{\mathrm{T} 4}\right)$. A three-factor latent-growth curve model was fit to the data to obtain estimates of intercept, linear change, and exponential change (for details, see methods in the supplementary materials). The fit of the model was acceptable, comparative fit index $(\mathrm{CFI})=0.986$, root mean square error of approximation $(\mathrm{RMSEA})=0.099$. Although reliable individual differences in $\mathrm{cRE}$ were present at baseline, these differences were completely wiped out by individual differences that emerged during the observation period (Fig. 2C). The correlation between $\mathrm{cRE}$ and neurons labeled with BrdU and neuronal marker NeuN did not differ significantly from zero at baseline, $r=0.24(P=$ $0.144)$. In contrast, at the end of the experiment,

\section{Fig. 1. Experimental setup and ef-} fects on body and brain weight. (A) Schematic illustration of the large enrichment enclosure housing 40 mice including RFID antenna positions (shown as red rings). Positions of levels, water sources, nesting boxes, and connecting tubes are drawn to scale. (Inset) Schematic illustration of animal tracking; an RFID passive integrated transponder (PIT) is implanted in mouse's neck. The electromagnetic field issued by the antenna induces the PIT to emit the number identifying the animal. This information is then picked up by the antenna and stored into a database together with spatial and temporal annotations. (B) Experimental time line. (C) Body weight development: weights (in grams) of CTR (blue) and ENR (red) mice at the beginning and end of the experiment. (D) Brain weights at perfusion (in grams). The difference in variance between CTR and ENR missed conventional statistical significance at $P=0.057$.
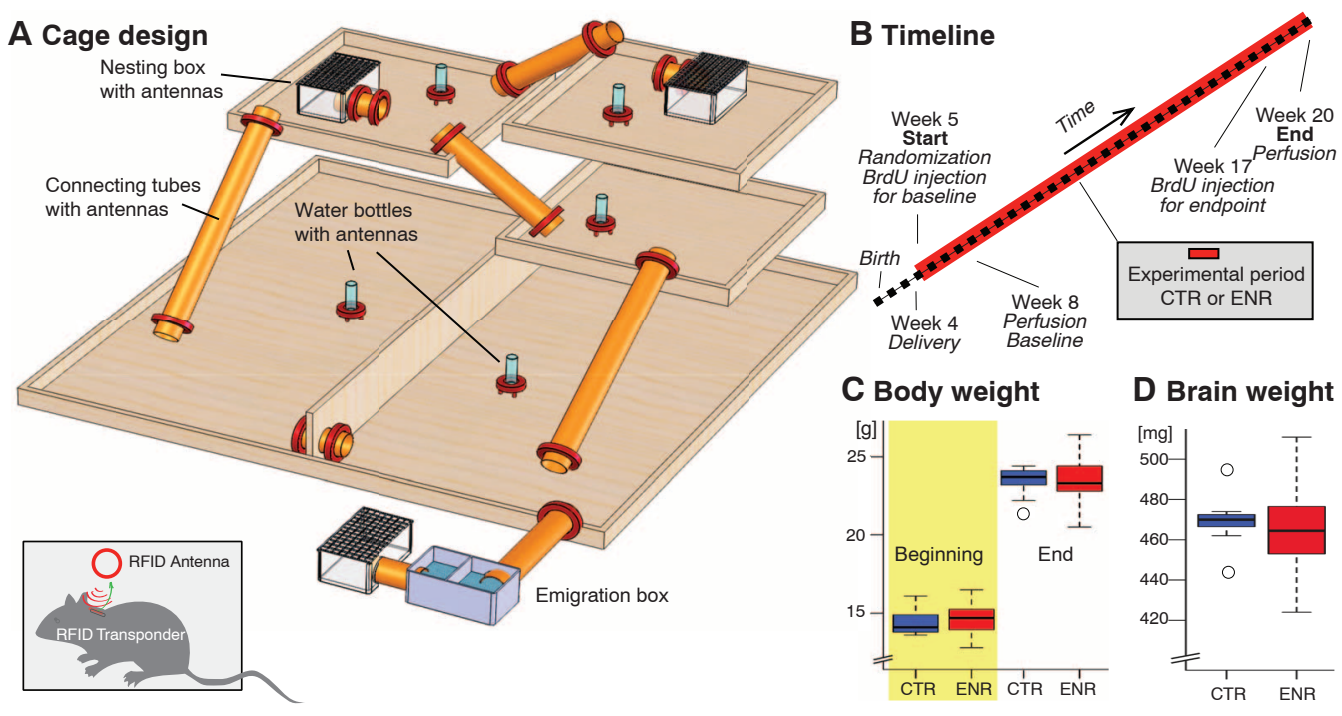

C Body weight 
the number of new neurons (BrdU and NeuN double-positive cells) correlated significantly with cRE at T4, $r=0.46(t=3.227, P=0.0026)$. Mice who explored their habitat more broadly also grew more new neurons in the hippocampus (Fig. 2D). An estimate of distance traveled (i.e., number of unique, nonrepetitive antenna contacts over the period of the experiment) showed a weaker but still significant association with adult neurogenesis $(r=$ $0.345 ; t=2.268 ; P=0.029)$, which explained $12 \%$ of the variance (fig. S2 and related information).

This study shows that adult neurogenesis, as an instantiation of brain plasticity, is linked to individual differences in experience among genetically identical individuals who live in a nominally identical environment. About one-fifth of the experiential effects on adult neurogenesis was captured by a measure of roaming through an enriched environment. This finding supports the idea that the key function of adult neurogenesis is to shape hippocampal connectivity according to individual needs and thereby to improve adaptability over the life course and to provide evolutionary advantage $(11,14,15)$. The observed individual differences in behavioral trajectories were reliable. This is in line with the observation that behavioral traits can be strongly influenced by external stimuli that vary between individuals or populations of individuals, as evidenced by diverging results of behavioral testing across different laboratories $(16,17)$.

The molecular mechanisms driving individual differences in behavioral and neural plasticity await further study. From the present results, three routes seem worth pursuing: (i) As full inbreeding is impossible, minimal residual segregation remains, and ontogeny may amplify the functional consequences of this residual genetic variation. However, this variability due to novel mutations corresponds to only 8 to 12 single-nucleotide polymorphisms across the entire genome (18). In addition, inbred mice might possibly genetically vary with respect to variable number tandem repeats and transposon insertions $(19,20)$ (ii) Stochastic gene regulation may lead to individual differences in molecular states, which are further amplified through experience. (iii) Animals might show small changes in the epigenetic state of their genome and may drift epigenetically apart over time, which reflects the cumulative effects of the choices they make in the course of their lives. This last explanation would be particularly in line with Turkheimer's argument (2) and consistent with data from human monozygotic twins, according to which epigenetic differences increase from young adulthood to old age and contribute to a growing discordance of monozygotic twins with advancing age (21). In addition, Lathe has suggested the following sources of initial individuality in rodents (19): intrauterine position, nutrition and interaction, imprinting errors, maternal stress and disease, and early postnatal interactions (including handling). Presumably, all of these would result in differences in the epigenome. When we obtained the animals for our study, we received mice randomly picked from as many litters as possible to achieve the best possible randomization.

As foreshadowed by psychological (22) and neurobiological (23) theories of ontogenetic development and in line with general theories of neural self-organization (24), small perturbations, possibly related to the factors mentioned above, may lead to initial individual differences in action tendencies. These differences, in turn, may trigger differences in experience that accumulate over time, result in differential plasticity, and correspond to different epigenetic states and developmental trajectories
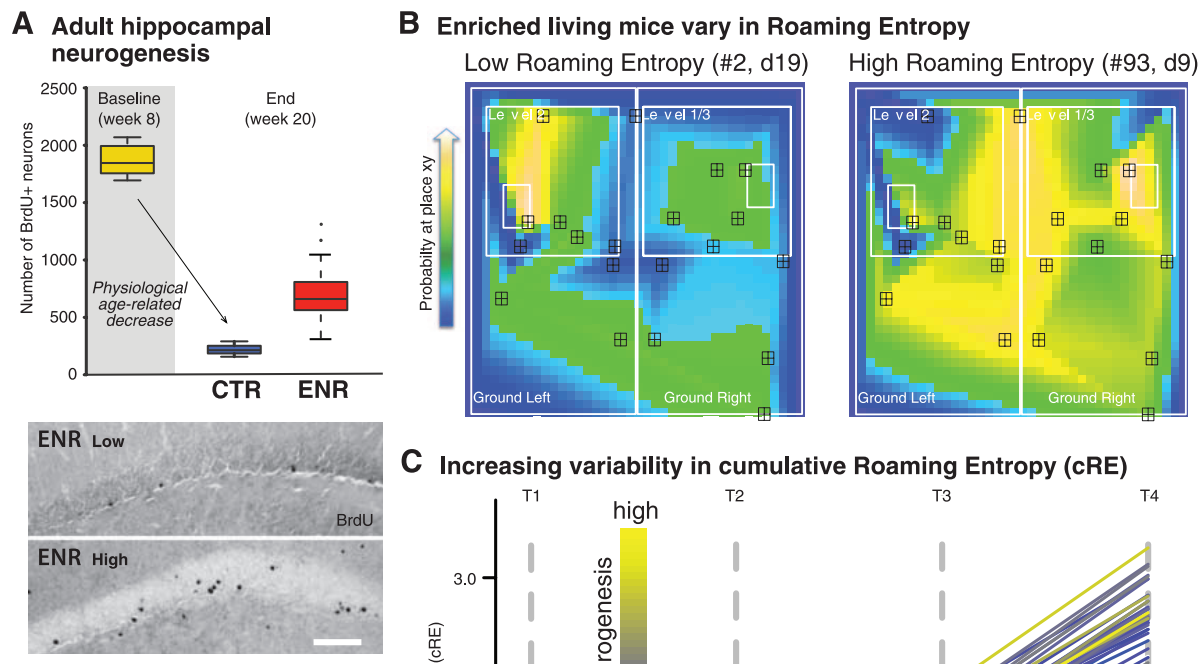

C Increasing variability in cumulative Roaming Entropy (cRE)
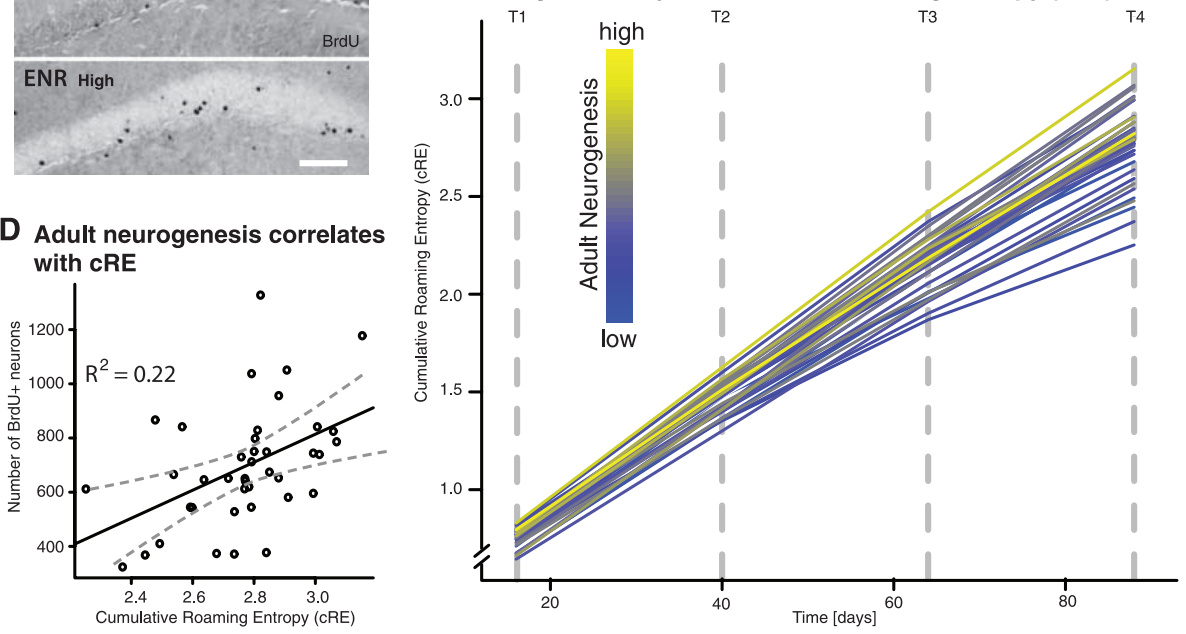

Fig. 2. Adult hippocampal neurogenesis and RE. (A) Total number of new neurons (BrdU+ and $\mathrm{NeuN}+$ cells) in the hippocampal dentate gyrus. Both CTR and ENR groups show the typical age-related decrease in neurogenesis. Compared with CTR mice, ENR mice increased adult hippocampal neurogenesis and resulted in a relative increase in variability. (Baseline versus CTR: $P=5.82 \times 10^{-9}$; baseline versus ENR: $P=7.17 \times 10^{-12}$, CTR versus ENR: $P=7.68 \times 10^{-5}$.) Exemplary histological images. (Top) "ENR low" shows the dentate gyrus of an animal with an adult neurogenesis level in the range of CTR, whereas the mouse in "ENR high" is at the upper end of neurogenesis levels in ENR. Scale bar, $150 \mu \mathrm{m}$. All analyses were done on continuous data, not by classifying animals into categories of high or low neurogenesis. (B) Exemplary heat maps of the RE of two mice in the large ENR enclosure (see also supplementary materials). The two panels are heat maps of explorative behavior for two mice assessed during one night depicting the probability of a mouse being in a specific location when viewed from above (i.e., aggregating across the four levels of the cage). Low probabilities are shown in blue, medium probabilities in green, and high probabilities in orange (see arrow on the left). Antenna positions are shown in black. (Left) A mouse with low RE (animal no. 2 at day 19); (right) a mouse with high RE (animal no. 93 at day 9). All analyses were done on continuous data, not by classifying animals into categories of high or low RE. (C) Measurements of RE were aggregated into four adjacent time periods to obtain an index of cumulative RE (CRE). Each line displays the CRE for a single mouse. Corresponding levels of neurogenesis are continuously color-coded from low (blue) to high (yellow). For exact values, see also the scatterplots in Fig. 2D. The mice differed in CRE at $\mathrm{T} 1$ (variance $\mathrm{T} 1=0.001, \chi^{2}=28.91, \mathrm{df}=1, P<0.0001$ ). At the same time, they differed markedly in rates of linear change $\left(\chi^{2}=18.76, \mathrm{df}=1, P<0.0001\right)$ and exponential change $\left(\chi^{2}=27.80, \mathrm{df}=1, P<\right.$ 0.0001 ). As a result, the variance in CRE at T4 (variance T4 $=0.022, \chi^{2}=35.12$ ) increased by a factor of 22 relative to the variance in CRE at T1 (for the difference, $\chi^{2}=31.73, \mathrm{df}=1, P<0.0001$ ). Individual differences in linear change predicted individual differences in CRE at the end of the observation period, $r=$ $0.98\left(\chi^{2}=118.742, P<0.0001\right)$, whereas individual differences in CRE at the beginning of the observation period did not predict individual differences in linear change $\left(\chi^{2}=3.19, P=0.074\right)$. (D) Individual differences in CRE are associated with individual differences in adult hippocampal neurogenesis. The number of new neurons correlated significantly with $\mathrm{CRE}$ at T4, $r=0.46(t=3.227, P=0.0026)$. 
Environmental enrichment does not seem generally to increase variability, although some controversy exists with regard to parameters such as body weight $(17,25,26)$. Most studies, however, have followed smaller cohorts of animals over shorter periods of time than in our study. Whether long-term enrichment in large groups and seminaturalistic conditions have a general varianceincreasing effect across a wide range of parameters remains to be determined.

Three months of living in a complex environment led to a massive magnification of individual differences in explorative behavior among genetically identical individuals over time, and these differences were related to adult hippocampal neurogenesis. The rich environment lost its "sameness" over time and gave way to the emergence of a personalized "life space" (27) and a "mouse individuality," similar to what has been observed in humans for personality traits (28). Hence, the magnitude of individual differences observed in replications of this experiment is likely to vary across studies: As the members of each new cohort individualize, their "society" will also be shaped in a slightly different, individual way. The present paradigm serves as an animal model for addressing the "mystery and controversy" (2) of the nonshared environment, or the ways in which living our lives makes us who we are (29).

\section{References and Notes}

1. P. C. M. Molenaar, D. I. Boomsma, C. V. Dolan, in Problems and Methods in Longitudinal Research: Stability and Change, D. Magnusson, G. Bergman, B. Rudinger, B. Törstad, Eds. (Cambridge Univ. Press, Cambridge, 1991), pp. 250-273.

2. E. Turkheimer, Psychol. Bull. 137, 825 (2011).

3. M. R. Rosenzweig, E. L. Bennett, Behav. Brain Res. 78, 57 (1996).

4. H. van Praag, G. Kempermann, F. H. Gage, Nat. Rev. Neurosci. 1, 191 (2000).

5. A. E. Whimbey, V. H. Denenberg, Behaviour 29, 296 (1967).

6. L. Lewejohann, B. Zipser, N. Sachser, Dev. Psychobiol. 53, 624 (2011).

7. G. Kempermann, Trends Neurosci. 31, 163 (2008).

8. L. Lewejohann et al., Behav. Res. Methods 41, 850 (2009).

9. B. Steiner et al., Glia 46, 41 (2004).

10. A. Garthe, J. Behr, G. Kempermann, PLoS ONE 4, e5464 (2009).

11. G. Kempermann, Nat. Rev. Neurosci. 13, 727 (2012).

12. G. Kempermann et al., Front Neurosci 4, 189 (2010).

13. K. Fabel et al., Front. Neurosci. 3, 50 (2009).

14. I. Amrein, H. P. Lipp, Biol. Lett. 5, 141 (2009).

15. G. Kempermann, J. Neurosci. 22, 635 (2002).

16. J. C. Crabbe, D. Wahlsten, B. C. Dudek, Science 284 1670 (1999).

17. L. Lewejohann et al., Genes Brain Behav. 5, 64 (2006).

18. D. W. Bailey, Immunol. Today 3, 210 (1982).

19. R. Lathe, Genes Brain Behav. 3, 317 (2004).

20. C. Julier et al., Proc. Natl. Acad. Sci. U.S.A. 87, 4585 (1990).

21. R. P. Talens et al., Aging Cell 11, 694 (2012).
22. P. B. Baltes, J. R. Nesselroade, S. W. Cornelius, Multivariate Behav. Res. 13, 127 (1978).

23. M. E. Raijmakers, P. C. Molenaar, Dev. Sci. 7, 149 (2004)

24. K. Friston, M. Breakspear, G. Deco, Front. Comput. Neurosci. 6, 44 (2012).

25. H. A. Van de Weerd et al., J. Appl. Anim. Welf. Sci. 5, 87 (2002).

26. D. P. Wolfer et al., Nature 432, 821 (2004).

27. K. Lewin, Dynamic Theory of Personality (McGraw-Hill, New York, 1935).

28. K. L. Jang, R. R. McCrae, A. Angleitner, R. Riemann, W. J. Livesley, J. Pers. Soc. Psychol. 74, 1556 (1998).

29. P. C. Molenaar, D. I. Boomsma, C. V. Dolan, Behav. Genet. 23, 519 (1993).

Acknowledgments: This study was financed from basic institutional funds. J.F. and I.K. were fellows of the International Max Planck Research School on the Life Course (LIFE), Berlin. M.K. has been supported by the International Research Training Group on Semantic Integration of Geospatial Information, funded by the German Research Foundation (DFG). The authors thank $S$. Vogler for drawing the cage in Fig. $1 A$ and D. Lasse for technical support. G.K. and U.L. would also like to thank ]. Nesselroade for inspiring discussions that helped shape the ideas presented in this report.

Supplementary Materials

www.sciencemag.org/cgi/content/full/340/6133/756/DC1

Materials and Methods

Figs. $\mathrm{S} 1$ and $\mathrm{S} 2$

References (30-35)

Movies $\mathrm{S} 1$ and $\mathrm{S} 2$

17 January 2013; accepted 22 March 2013

10.1126/science.1235294

\section{Compartmentalization of GABAergic Inhibition by Dendritic Spines}

Chiayu Q. Chiu, ${ }^{1,2 *}$ Gyorgy Lur, ${ }^{1,2 *}$ Thomas M. Morse, ${ }^{1}$ Nicholas T. Carnevale, ${ }^{1}$ Graham C. R. Ellis-Davies, ${ }^{3}$ Michael ]. Higley ${ }^{1,2} \dagger$

$\gamma$-aminobutyric acid-mediated (GABAergic) inhibition plays a critical role in shaping neuronal activity in the neocortex. Numerous experimental investigations have examined perisomatic inhibitory synapses, which control action potential output from pyramidal neurons. However, most inhibitory synapses in the neocortex are formed onto pyramidal cell dendrites, where theoretical studies suggest they may focally regulate cellular activity. The precision of GABAergic control over dendritic electrical and biochemical signaling is unknown. By using cell type-specific optical stimulation in combination with two-photon calcium $\left(\mathrm{Ca}^{2+}\right)$ imaging, we show that somatostatin-expressing interneurons exert compartmentalized control over postsynaptic $\mathrm{Ca}^{2+}$ signals within individual dendritic spines. This highly focal inhibitory action is mediated by a subset of GABAergic synapses that directly target spine heads. GABAergic inhibition thus participates in localized control of dendritic electrical and biochemical signaling.

A challenge to elucidating the function of synaptic inhibition is the diversity of $\gamma$ aminobutyric acid-releasing (GABAergic) interneurons found in cortical circuits $(1-3)$. Several interneuron classes, including those that express somatostatin (SOM-INs), target the dendrites of excitatory, glutamatergic pyramidal cells (3-5). SOM-INs regulate the initiation of action potential bursts generated via active currents in postsynaptic dendrites $(6-8)$. We hypothesized that these inputs might also exert focal influence over dendritic signaling. Here, we used electrophysiological, optical, and computational approaches to investigate the localized actions of GABAergic inhibition in pyramidal cell dendrites.

To activate dendritic GABAergic synapses, we used a somatostatin-Cre mouse line (9) (Fig. 1A and fig. S1, A and B) to conditionally express channelrhodopsin-2 (ChR2) (10) in SOM-INs of the prefrontal cortex (fig. S1, C and D). In acute brain slices prepared 2 to 3 weeks after viral injection, pulses of light $(5 \mathrm{~ms}, 473 \mathrm{~nm})$ delivered through the microscope objective evoked action potentials (APs) in fluorescently identified SOMINs (fig. S2, A to C). Whole-cell recordings in layer $2 / 3$ pyramidal neurons revealed corresponding inhibitory postsynaptic potentials (IPSPs) sequent experiments, type A GABA receptor $\left(\mathrm{GABA}_{\mathrm{A}} \mathrm{R}\right)$-mediated IPSPs were isolated by including the selective type $\mathrm{B}$ GABA receptor $\left(\mathrm{GABA}_{\mathrm{B}} \mathrm{R}\right)$ antagonist CGP-55845 in the perfusate (Fig. 1C). IPSPs exhibited a reversal potential of $-69.9 \pm 1.5 \mathrm{mV}($ mean $\pm \mathrm{SEM}, n=5)$ (Fig. 1, B and C, and fig. S2, D to F). For sub- that did not differ significantly from the value recorded via gramicidin-based perforated patch $(-72.4 \pm 1.7, n=6, P=0.3$, fig. S2, G and $\mathrm{H})$.

To determine how inhibition influences dendritic activity in pyramidal neurons, we used twophoton laser scanning microscopy (2PLSM) to image calcium $\left(\mathrm{Ca}^{2+}\right)$ in apical dendritic spines and shafts. $\mathrm{Ca}^{2+}$ transients $\left(\Delta \mathrm{Ca}^{2+}\right)$ were evoked by somatic APs (Fig. 1, D and E, and fig. S3, A and $\mathrm{B}$ ) and were mediated by voltage-gated $\mathrm{Ca}^{2+}$ channels (VGCCs) (fig. S3C). We compared APevoked $\mathrm{Ca}^{2+}$ signals under control conditions $\left(\Delta \mathrm{Ca}^{2+}{ }_{\mathrm{ctt}}\right)$ and when preceded by an IPSP $\left(\Delta \mathrm{Ca}^{2+}{ }_{\text {inh }}\right)$ (15-ms interval) evoked by a light pulse targeting the imaged region (Fig. 1E). In 57\% (73/127) of randomly imaged spines, optical activation of SOM-INs produced a significant reduction $(>15 \%$, see methods and fig. S3, D to F) in the AP-evoked $\Delta \mathrm{Ca}^{2+}$. At these locations, the average $\mathrm{Ca}^{2+}$ inhibition $\left(\Delta \mathrm{Ca}^{2+}{ }_{\text {inh }} / \Delta \mathrm{Ca}^{2+}{ }_{\text {ctt }}\right)$ was significantly greater for spines than for neighboring dendritic shafts $(0.60 \pm 0.02$ versus $0.78 \pm 0.03$, $P<0.001$; Fig. 1, F and G). The inhibition of $\Delta \mathrm{Ca}^{2+}$ was abolished by application of the $\mathrm{GABA}_{\mathrm{A}}$ antagonist picrotoxin $(n=8, P<0.05$, Fig. $1 \mathrm{H})$. Similar $\mathrm{Ca}^{2+}$ inhibition was seen in basal dendrites (23/49 spines; $0.73 \pm 0.02$ versus $0.87 \pm 0.02$ for spines and shafts, respectively, $P<0.01$; fig. S4).

${ }^{1}$ Department of Neurobiology, Yale School of Medicine, New Haven, CT 06510, USA. ² Yale Program in Cellular Neuroscience, Neurodegeneration, and Repair, Yale School of Medicine, New Haven, CT 06510, USA. ${ }^{3}$ Department of Neuroscience, Mount Sinai School of Medicine, New York, NY 10029, USA.

*These authors contributed equally to this work. †Corresponding author. E-mail: m.higley@yale.edu 